\title{
VIRTUAL SCREENING OF INDONESIAN HERBAL DATABASE FOR CP ALLOSTERIC MODULATOR OF HEPATITIS B VIRUS
}

\author{
ULFA IVONIE ${ }^{1}$, ARRY YANUAR ${ }^{1 *}$, FIRDAYANI $^{2}$ \\ ${ }^{1}$ Department of Biomedical Computation Faculty of Pharmacy, Universitas Indonesia, Depok, 16424, Indonesia. ${ }^{2}$ Center for \\ Pharmaceutical and Medical Technologies, Agency for Assessment and Application of Technology, Jakarta, 10340, Indonesia. \\ Email: arry.yanuar@ui.ac.id
}

Received: 23 May 2018, Revised and Accepted: 25 September 2018

ABSTRACT

Objective: This study performed a virtual screening of the Indonesian Herbal Database for the core protein allosteric modulator of the hepatitis B virus (HBV) using AutoDock and AutoDock Vina software, to discover novel safe drugs for patients.

Methods: The method was validated using the parameters enrichment factor (EF), receiver operating characteristics, and area under the curve (AUC). The grid box size used in virtual screening with AutoDock was $55 \times 55 \times 55$ with EF10\% of 0.7652 and AUC of 0.6709 , whereas that used in virtual screening with AutoDock Vina was $20.625 \times 20.625 \times 20.625$ with EF5\% of 0.5075 and AUC of 0.7832 .

Results: The top 10 compounds from virtual screening with AutoDock at G levels -11.74--10.31 kcal/mol were yuehchukene, lansionic acid, stigmast4-en-3-one, myrtillin, sanggenol 0, lanosterol, erycrista-gallin, alpha-spinasterol, cyanidin 3-arabinoside, and cathasterone and with AutoDock Vina at G levels -12.1 to $-10.7 \mathrm{kcal} / \mathrm{mol}$ were sanggenol 0, cucumerin A, yuehchukene, palmarumycin CP1, dehydrocycloguanandin, myrtillin, liriodenine, myricetin 3-alpha-L-arabinopyranoside, myricetin 3-galactoside, and cassameridine.

Conclusion: Three compounds were in top list of both virtual screening methods against Cp allosteric modulator of HBV are myrtillin, sanggenol O, and yuehchukene have a prospect to be investigated futher for anti HBV.

Keywords: Hepatitis B virus, Core protein, Cp allosteric modulator, Virtual screening, Indonesian herbal medicine.

(C) 2018 The Authors. Published by Innovare Academic Sciences Pvt Ltd. This is an open access article under the CC BY license (http://creativecommons. org/licenses/by/4. 0/) DOI: http://dx.doi.org/10.22159/ijap.2018.v10s1.42

\section{INTRODUCTION}

Hepatitis B is an inflammation of the liver caused by the hepatitis B virus (HBV). HBV is transmitted hematogenously and sexually, causing a complicated viral-host interaction which results in either an acute symptomatic disease or an asymptomatic disease [1]. Hepatitis B is the most serious type of viral hepatitis and one of the major problems of global public health [2]. Among countries in Southeast Asia, Indonesia has the second highest prevalence of hepatitis B. Based on Indonesian Basic Health Research in 2013, 100 Indonesian blood donors were tested, and 10 were found to have hepatitis B or C infection. Therefore, it can be estimated that around 28 million Indonesians are infected with hepatitis B or C, out of which 14 million may be expected to experience chronic disease, and of these, 1.4 million are likely to be diagnosed with liver cancer in the future. The drugs that are currently approved by the FDA and used to treat hepatitis B infection in other countries consist of two classes: Nucleotide analogs and interferon alpha. However, these two classes of drugs both have disadvantages. In the interferon alpha group, drugs are administered parentally and can cause adverse effects such as myalgia, nausea, myelosuppression, leukopenia, and anorexia. Interferon alpha drugs are also contraindicated in patients with cirrhotic decompensation or hepatitis exacerbations and patients with autoimmune or psychiatric disease. Nucleotide analogs also have side effects such as nausea, fatigue, upper abdominal pain, and upper respiratory infections, and their long-term use can lead to drug resistance. Therefore, further research is needed to discover novel safe drugs for patients. One method for doing this is in silico, as we describe here.

The computational methodology has been crucial for the development of drug discovery from the identification of lead compounds, ligandbased approaches or structures, to molecular docking, and virtual screening. These techniques are widely used in new drug discovery efforts because they can save research costs and time. Therefore, computational methods are appropriate for attempting to discover new drugs to combat hepatitis B. Core proteins (Cps) can be used as targets for hepatitis B drugs because there are no HBV Cp homologs in humans, and the $\mathrm{Cp}$ is crucial to every stage of the viral life cycle [3]. One of the drug classes that target $\mathrm{Cp}$ is the $\mathrm{Cp}$ allosteric modulator (CpAM). This drug class affects the subunit structure of $\mathrm{Cp}$ to prevent the formation of nucleocapsids and alter cccDNA function.

\section{MATERIALS AND METHODS}

\section{Methods}

The hardware consists of three computers and a laptop that had different specifications. (CPU) Intel® Xeon E5620, Nvidia ${ }^{\circledR}$ FeForce GTX 780 graphics processing unit (GPU), and 32 Gigabyte (GB) DDR3 random access memory (RAM) were used. The second computer had an Intel Core 2 Quad CPU, Nvidia ${ }^{\circledR}$ GeForce 9400 GT GPU, and 2 GB of DDR2 RAM. The third computer had an Intel® Core i5 $2.50 \mathrm{GHz}$ CPU, integrated GPU, and 6GB of DDR3 RAM. The main and secondary computers had the Ubuntu Linux operating system 12.04 LTS. The tertiary computer is MacMini with MacOS Yosemite operating system. The laptop was privately-owned Acer ${ }^{\circledR}$ E1-410 which features the Intel ${ }^{\circledR}$ Celeron $₫$ quad-core processor N2920 (2M Cache, $2.0 \mathrm{GHz}$ ), RAM of 2 GB DDR3, with Windows 864 bit operating system.

The analysis was performed using the following software: AutoDock Tools, PyRx, AutoDock, AutoDock Vina, Open Babel, PyMol, Marvin Sketch, Chimera, and Ligand Scout. The analysis was performed on the three-dimensional structure of the target protein macromolecule 5WRE, obtained from the Research Collaboratory for Structural Bioinformatics Protein Data Bank (http://www.rcsb.org/pdb) with 
a resolution of $1.95 \AA$. A total of 1411 three-dimensional structures of herbal compounds were obtained from the Indonesian Medicinal Plant Database (http://herbaldb.farmasi.ui.ac.id). A total of 39 threedimensional structures of positive control compounds were obtained by manual drawing using Marvin Sketch. A total of 1400 three-dimensional structures of a static control compound (decoy) were obtained from the DUD-E website page (dude.docking.org).

Macromolecules were separated from their cocrystal ligands using AutoDock Tools to obtain the major protein chains. From these protein chains, the selected chains to be used as tethers were stored as.pdb files. Optimization of macromolecular structures was done using AutoDock Tools. As part of this optimization, a water molecule was removed, a polar hydrogen molecule was added and merged with a nonpolar hydrogen molecule, and a Gasteiger charge was added. After the optimization, the results obtained were stored as a pdbqt file. Ligand compounds consisting of positive controls were drawn in Marvin Sketch in 2D, then converted into three dimensional and stored as.pdb files. Negative control (decoy) was obtained from SMILES positive controls uploaded to the DUD-E website (dude.docking.org). Then, the ligands were docked to the macromolecule using AutoDock and AutoDock Vina.

Validation was performed using AutoDock and AutoDock Vina software by molecular docking. The validation results consisted of enrichment factor (EF), receiver operating characteristic (ROC), and area under the curve (AUC). When performing the existing tethering, parameters were varied to identify which those that produced the best leaching method. The software used for virtual screening was PyRx. Inside the application were AutoDock, AutoDock Vina, and Open Babel. Compounds obtained from the Herbal DB database in.mol2 format were first converted into.pdbqt format using the Open Babel software and subsequently tethered to the target macromolecular site. Tethering results obtained from AutoDock and AutoDock Vina were stored as.pdb files, which can be visualized using PyMOL, Chimera, or LigandScout. Visualization showed the superposition of the ligand as well as the interactions between macromolecules and ligands.

\section{RESULTS AND DISCUSSION}

\section{Macromolecular chain determination}

The initial cocrystal ligand that previously separated from the macromolecule was tethered back to the macromolecule. The central bond between ligand and macromolecule was placed at the center of the ligand so that the coordinate points obtained were $X=-29.944$, $\mathrm{Y}=-9.723$, and $\mathrm{Z}=-6.669$, with dimensions of $40 \AA$ on each axis. Then, the root mean square deviation (RMSD) value was calculated between the initial ligand and the ligand of the complex after recombination.

The lowest RMSD value was obtained by the $\mathrm{BC}$ chain with a value of $0.2107 \AA$ and an energy binding value $(\Delta G)$ of -10.97 , where the chain was the dimer interface of the $\mathrm{AB}$ and $\mathrm{CD}$ chains (Table 1). The dimer interface chain was used because the active ligand site area has a large mooring range of operations that can work between two chains, weaken the interaction between dimers, induce conformational changes in $\mathrm{HBc}$, and make the capsid unstable so that it cannot be formed [4].

Table 1: Data resulting from redocking ligand to the macromoleculess

\begin{tabular}{llll}
\hline Chain's name & Ligand & $\boldsymbol{\Delta G}(\mathbf{k c a l} / \mathbf{m o l})$ & RMSD $(\AA)$ \\
\hline A & 7TL & -7.19 & 0.6534 \\
B & 7TL & -7.36 & 0.5691 \\
C & 7TL & -7.44 & 0.5025 \\
D & 7TL & -7.57 & 0.5648 \\
E & 7TL & -7.60 & 0.6398 \\
F & 7TL & -7.97 & 0.5903 \\
AF & 7TL & -11.49 & 0.7581 \\
BC & 7TL & -10.97 & 0.2107 \\
DE & 7TL & -11.04 & 0.7778
\end{tabular}

\section{Grid box optimization}

Optimization of the box was performed by tethering the existing ligand with the macromolecule that had been selected, commonly called redocking. Docking was performed on different box sizes ranging from $30 \times 30 \times 30$ to $105 \times 105 \times 105$ at intervals of 5 , then 100 runs were performed. The center of the bond between ligand and macromolecule was placed at the center of the ligand. The coordinate point obtained was $\mathrm{X}=-29.944, \mathrm{Y}=-9.723$, and $\mathrm{Z}=-6.669$ using AutoDock inside PyRx. From the redocking results, the sizes of five boxes were obtained (Table 2).

\section{Validation of virtual screening methods with autodock $E F$}

EF was calculated by comparing the active compound obtained with the expected active compound based on the available data. The EF value indicates the accuracy of virtual screening, with a good value being close to 1 .

Calculation of EF values was performed at 1\%, 5\%, 10\%, and 20\%. On the basis of data obtained at EF1\% (19 first ligands) and EF5\% (97 first ligands), there was no positive control emergence on all grid boxes. On the grid box with size $65 \times 65 \times 65$, a new EF value appeared at $20 \%$. This suggested that the positive control compound was detected longer than the decoy, indicating that the positive control did not interact strongly with macromolecules compared with the decoy. One of the causes for this could be the sensitivity of the tools on tethering of the compound with the macromolecule. At EF $10 \%$, the highest value was obtained with the grid box with size $55 \times 55 \times 55$ with a value of 0.7652 and the lowest value obtained with the grid box with size $65 \times 65 \times 65$ with a value of 0 , showing that the positive control did not appear in the first 194 ligands. The highest value of EF was obtained with the grid box with size $45 \times 45 \times 45$ at EF20\% with a value of 1.0256 , but at EF10\%, the value obtained was only 0.2524 . For the grid box with size $55 \times 55 \times 55$, EF10\% and EF20\% values obtained were 0.7652 (Table 3).

\section{ROC}

The ROC curve was obtained on the basis of data from the ligand control results of positive and decoy controls. The obtained data were sorted from the least to the highest binding energy value. Then, true positive

Table 2: Data binding energy and RMSD tethering back 5WRE

\begin{tabular}{lll}
\hline Grid Box & Binding energy/G (kcal/mol) & RMSD (Å) \\
\hline $30 \times 30 \times 30$ & -11.64 & 0.5422 \\
$35 \times 35 \times 35$ & -11.62 & 0.5711 \\
$40 \times 40 \times 40$ & -11.62 & 0.6392 \\
$45 \times 45 \times 45$ & -11.60 & 0.5609 \\
$50 \times 50 \times 50$ & -11.55 & 0.7688 \\
$55 \times 55 \times 55$ & -11.55 & 0.5271 \\
$60 \times 60 \times 60$ & -11.63 & 0.5690 \\
$65 \times 65 \times 65$ & -11.64 & 0.5540 \\
$70 \times 70 \times 70$ & -11.58 & 0.7698 \\
$75 \times 75 \times 75$ & -11.45 & 0.7151 \\
$80 \times 80 \times 80$ & -11.61 & 0.6033 \\
$85 \times 85 \times 85$ & -11.38 & 0.4883 \\
$90 \times 90 \times 90$ & -11.46 & 0.4926 \\
$95 \times 95 \times 95$ & -11.38 & 0.4521 \\
$100 \times 100 \times 100$ & -11.49 & 0.5700 \\
$105 \times 105 \times 105$ & -11.41 & 0.6485 \\
\hline
\end{tabular}

Table 3: EF calculation results using AutoDock

\begin{tabular}{lllll}
\hline Grid Box & EF1\% & EF5\% & EF10\% & EF20\% \\
\hline $45 \times 45 \times 45$ & 0 & 0 & 0.2524 & 1.0256 \\
$55 \times 55 \times 55$ & 0 & 0 & 0.7652 & 0.7652 \\
$65 \times 65 \times 65$ & 0 & 0 & 0 & 0.2524 \\
$75 \times 75 \times 75$ & 0 & 0 & 0.2524 & 0.2524 \\
$85 \times 85 \times 85$ & 0 & 0 & 0.2524 & 0.2524 \\
\hline
\end{tabular}


ratio (TPR), also called hit rate and recall, and the existing false positive ratio (FPR) was calculated. The ROC curve was obtained by comparing the TPR and FPR values. On the X-axis, FPR data were plotted, whereas on the Y-axis, TPR data were plotted. On the ROC curve, there was also a linear line random line to help assess the results obtained.

On the basis of the combined ROC curves (Fig. 1), none of the overall curves were above the random line, but the best curve line can be seen on the grid box with size $55 \times 55 \times 55$. After ROC curves were obtained, the value of AUC was calculated in two ways, that is, trapezoid and integral equations that started from linear regression equation of the integral ROC curve [5]. The best AUC value was for the grid box with size $55 \times 55 \times 55$ with a value of 0.6709 . For this grid box, the value of EF obtained close to 1 (0.7652); therefore, the size of the grid box used to perform virtual screening of Herbal DB compounds using AutoDock was $55 \times 55 \times 55$ (Table 4 )

\section{Validation of virtual screening methods with autodock Enrichment factor}

A value of 0 was obtained at EF1\% for different grid box sizes (Table 5). This suggests that the method used was less sensitive or the positive control compound did not strongly interact with macromolecules compared with the decoy so that when the decoy plating was tethered earlier than the positive control compound. At EF5\%, only two grid box sizes produced results: $16.875 \times 16.875 \times 16.875$ had a value of 1.0256 and $20.625 \times 20.625 \times 20.625$ had a value of 0.5075 . At EF10\%, the highest value of 3.4991 was obtained on the grid box with size $16.875 \times 16.875 \times 16.875$, but at EF20\%, the value obtained decreased to 2.5085 . At EF $20 \%$, the highest value obtained on the grid box with size $20.625 \times 20.625 \times 20.625$ was 3.0700 , and the lowest value obtained on the grid box with size $31.875 \times 31.875 \times 31.875$ was 0.7652 .

\section{ROC}

Examining the combined ROC curves (Fig. 2), there was no curve that was entirely above the random line, but the best curve was seen on the $20.625 \times 20.625 \times 20.625$ grid box, with the curve almost entirely above the random line. The area under the ROC curve is called the AUC

The best AUC value of 0.7832 was found on the grid box $20.625 \times 20.625 \times 20.625$, indicating that there was a good separation

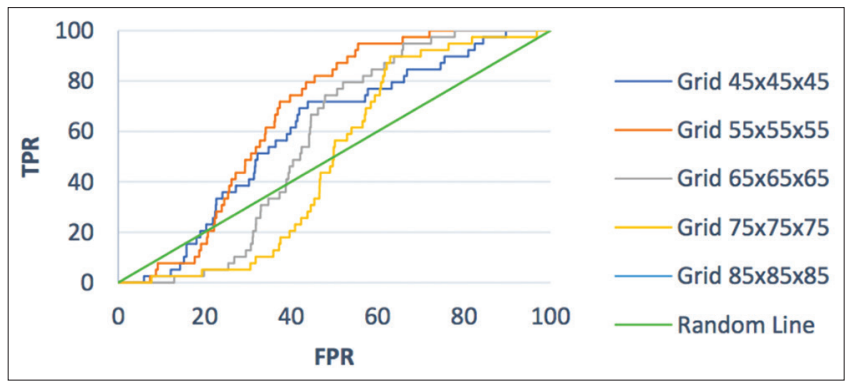

Fig. 1: Combined receiver operating characteristic curves of validation results using AutoDock

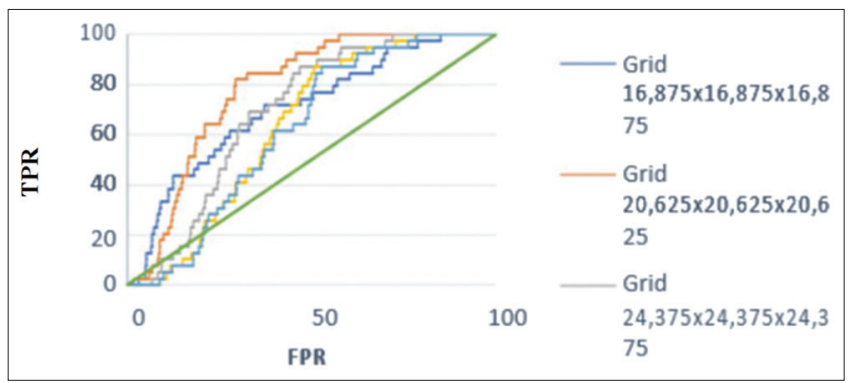

Fig. 2: Combined receiver operating characteristic curve validation results using AutoDock Vina between the active compound and inactive compound. In addition, to get a good AUC value, the EF value on the grid box was also good, so the grid box size of $20.625 \times 20.625 \times 20.625$ can be used to perform virtual screening of Herbal DB compounds using AutoDock Vina (Table 6)

\section{Virtual screening}

Virtual screening using AutoDock

Virtual screening using AutoDock was performed with PyRx. The parameters used were the grid box size of $55 \times 55 \times 55$ because, on the basis of the EF and AUC values that were obtained, this grid box size had the best value. The central coordinates used centered on the ligand with the coordinate point $\mathrm{X}=-29.944, \mathrm{Y}=-9.723$, and $\mathrm{Z}=-6.669$ using $\mathrm{Ga}$ run 100. After screening 1411 compounds derived from the Indonesian medicinal plant database (Herbal DB), the 10 best compounds with the smallest binding energy value were selected (Table 7).

In the first position were yuehchukene compounds, obtained from the Murraya paniculata plant in the family Rutaceae. In Indonesia, this plant is known by the name of yellow. Conventionally, this plant is used to treat rheumatism, ulcers, bruises, and toothache. Lansionic acid was in the second position. This compound is found in the plant Lansium

Table 4: AUC value using AutoDock

\begin{tabular}{ll}
\hline Grid Box & AUC \\
\hline $45 \times 45 \times 45$ & 06021 \\
$55 \times 55 \times 55$ & 0.6709 \\
$65 \times 65 \times 65$ & 0.5694 \\
$75 \times 75 \times 75$ & 0.4920 \\
$85 \times 85 \times 85$ & 0.4823 \\
\hline AUC: Area under the curve
\end{tabular}

Table 5: EF calculation results using AutoDock Vina

\begin{tabular}{lllll}
\hline Grid Box & EF1\% & EF5\% & EF10\% & EF20\% \\
\hline $16.875 \times 16.875 \times 16.875$ & 0 & 1.0256 & 3.4991 & 2.5085 \\
$20.625 \times 20.625 \times 20.625$ & 0 & 0.5075 & 1.8237 & 3.0700 \\
$24.375 \times 24.375 \times 24.375$ & 0 & 0 & 1.0256 & 1.4215 \\
$28.125 \times 28.125 \times 28.125$ & 0 & 0 & 0.2524 & 0.8951 \\
$31.875 \times 31.875 \times 31.875$ & 0 & 0 & 0.5075 & 0.7652 \\
\hline
\end{tabular}

EF: Enrichment factor

Table 6: AUC values using AutoDock Vina

\begin{tabular}{ll}
\hline Grid box & AUC \\
\hline $16.875 \times 16.875 \times 16.875$ & 0.7054 \\
$20.625 \times 20.625 \times 20.625$ & 0.7832 \\
$24.375 \times 24.375 \times 24.375$ & 0.6952 \\
$28.125 \times 28.125 \times 28.125$ & 0.6379 \\
$31.875 \times 31.875 \times 31.875$ & 0.6234 \\
\hline
\end{tabular}

Table 7: Results of virtual screening of Indonesian herbal plants with AutoDock

\begin{tabular}{lllc}
\hline No & $\begin{array}{l}\text { Herbal DB } \\
\text { code }\end{array}$ & Compound name & $\begin{array}{l}\text { Binding energy/ } \\
\text { G (kcal/mol) }\end{array}$ \\
\hline 1 & M00000490 & Yuehchukene & -11.74 \\
2 & M00035124 & Lansionic acid & -11.01 \\
3 & M00029821 & Stigmast-4-en-3-one & -10.71 \\
4 & M00006698 & Myrtillin & -10.58 \\
5 & M00014245 & Sanggenol 0 & -10.58 \\
6 & M00003657 & Lanosterol & -10.52 \\
7 & M00010027 & Erycristagallin & -10.48 \\
8 & M00003673 & Alpha-spinasterol & -10.40 \\
9 & M00006650 & Cyanidin 3-arabinoside & -10.35 \\
10 & M00000199 & Cathasterone & -10.31 \\
\hline
\end{tabular}


domesticum, which in Indonesia is known as "naman duku." The resin is non-toxic and can be used as a medicine for diarrhea. Its bark has been used to treat dysentery and malaria, whereas its leaves are antiinflammatory. In the third position was the compound stigmast-4-en-3one, which can be obtained from Zea mays (or maize). A hepatitis B oral vaccine derived from this plant is being developed. In the fourth position was myrtillin compound. These compounds can be obtained from the plants Clitoria ternatea, Mangifera indica, Muntingia calabura, Punica granatum, and Vitis vinifera. C. ternatea is in the family Fabaceae and is known to have anti-inflammatory and antioxidant effects. $M$. indica is in the Anacardiaceae family and is known in Indonesia as mango. Mango has a variety of effects and properties including antidiabetes, antioxidant, antiviral, anti-inflammatory, antibacterial, antifungal, anthelmintic, antiparasite, antimicrobial, immunosuppressive, and hepatoprotective. Experiments have shown that mangoes effectively protect against oxidative stress that induces rat liver cells by modulating cell growth regulators. M. calabura, in the Elaeocarpaceae family, has antinociceptive activity involving peripheral and central nervous system activation, as well as partial modulation through opioid and NO/ cGMP receptor pathways. P. granatum, known in Indonesia as delima, is known to have antiviral and antibacterial properties and can play a role in fighting cancer cells. $V$. vinifera in Indonesia is known by the name of wine. This plant effectively reduces the secretion of HBsAg and HBeAg from HepG2.215 cells [6].

In the fifth position was the compound sanggenol 0 , which can be obtained from the plant Morus australis. This plant is in the Moraceae family and is known to have astringent, anthelmintic, and hypoglycemic effects. In the sixth position was lanosterol compounds, which can be obtained from the plant Euphorbia pulcherrima, a member of the family Euphorbiaceae. In Indonesia, this plant is known as "denok." Sap from this plant is used for hair removal, but it can cause allergic reactions for sensitive people and can cause irritation to the skin and stomach. If this plant is eaten, it can cause vomiting and diarrhea. In the seventh position was erycristagallin, a compound found in the plant Erythrina orientalis in the family Fabaceae. This plant in Indonesia is known by the name dadap and has antispasmodic, antioxidant, analgesic, anti-inflammatory, antifungal, and antibacterial effects. In the eighth position was alpha-spinasterol compound. These compounds can be obtained from Pisonia grandis and Platycodon grandiflorum. P. grandis is known for hepatoprotection that prevents the destruction of liver tissue of atrazine-induced fish. It has been demonstrated that adjuvant hepatitis B vaccine derived from $P$. grandiflorum significantly increases the potential activation of $\mathrm{T}$ and $\mathrm{B}$ cells in immunized HBsAg mice, modulating the quality of the immune response and resulting in a balanced Th1/Th2 immune response to the sensitivity-related HBsAgrelated interception levels of IgG2a, IgG2b, and IgG1 levels.

In the ninth position was 3-arabinoside cyanidin compounds, which can be obtained from the plants Cinchona succirubra, M. indica, and Theobrama cacao. C. succirubra, in the family Rubiaceae, is known to function as an antimalarial but can also be used as antiobecity. T. cacao of the family Sterculiaceae is known in Indonesia as the chocolate tree. It has been shown that the consumption of chocolate can reduce damage to blood vessels in patients with cirrhosis and also decrease blood pressure in the liver. Dark chocolate contains powerful antioxidants that reduce post-prandial (after-meal) blood pressure in the liver (or portal hypertension) associated with impaired liver blood vessels. In the tenth position was cathasterone. This compound can be obtained from Catharanthus roseus. On the basis of experiments, the flower extract from this plant can promote wound healing, and it has shown antimicrobial activity against Pseudomonas aeruginosa and Staphylococcus aureus.

\section{Virtual screening using AutoDock Vina}

Virtual screening using AutoDock Vina was performed on 1411 compounds derived from Herbal DB. Grid boxes used were of the size $20.625 \times 20.625 \times 20.625$ with central coordinates centered on the ligand at $\mathrm{X}=-29.944, \mathrm{Y}=-9.723$, and $\mathrm{Z}=-6.669$, and using ga run of 100 .
From 1411 compounds on which we performed virtual screening, 10 compounds with the best binding energy were selected (Table 8).

In the first position was sanggenol compound $\mathrm{O}$ and in the second position was cucumerin A, contained in the plant Cucumis sativum, commonly referred to as cucumbers in Indonesia. Research has shown that cucumber is an ideal plant for HBsAg expression to be used as an oral vaccine [7]. In third place was yuehchukene compound, and in the fourth place was palmarumycin CP1 compounds derived from the Jatropha curcas plant in Java (known as Jarak). The leaf extract of this plant has been shown to act as a malarial parasite inhibitor and to increase the phagocytic activity of tumoricidal macrophages [8]. Dehydrocycloguanandin compounds ranked fifth. These compounds can be obtained from the Calophyllolide inophylum plant, of the Clusiaceae family, the fruit of which is known to be anti-inflammatory [9]. Another plant containing dehydrocycloguanandin compounds is Mesua ferrea from the family Guttiferae. The leaf extract of this plant has shown immunosuppressive activity, while the flower extract has shown antioxidant effects and hepatoprotective activity [10]. The compound myrtillin occupied the sixth place.

In seventh place was liriodenine compounds, which can be obtained from the plants Annona reticulata, Nelumbo nucifera, and Polyalthia longifolia. A. reticulata comes from the Annonaceae family. This plant has been shown to have analgesic and anti-inflammatory effects, has shown antiproliferative activity, has been found to be useful for wound healing, and has antioxidant and antimicrobial properties. Besides, liriodenine is used both as topoisomerase I inhibitor and topoisomerase II inhibitor [11]. N. nucifera, known in Indonesia as lotus, has been shown to reduce the production of HBsAg. P. longifolia, of the Annonaceae family, is known by the name Mempisang in Indonesia and has been found to have anti-inflammatory and hepatoprotective effects. In the eighth position was a 3-alpha-L-arabinopyranoside myricetin compound that can be obtained from the Woodfordia fruticosa plant. As shown in experiments, the flower extract of this plant has hepatoprotective properties, and a histopathological analysis showed

Table 8: Results of virtual screening of Indonesian herbal plants with AutoDock Vina

\begin{tabular}{lllc}
\hline No. & $\begin{array}{l}\text { Herbal DB } \\
\text { code }\end{array}$ & Compound's name & $\begin{array}{c}\text { Binding } \\
\text { energy/ } \\
\text { G (kcal/mol) }\end{array}$ \\
\hline 1 & M00014245 & Sanggenol 0 & -12.1 \\
2 & M00014015 & Cucumerin A & -11.9 \\
3 & M00000490 & Yuehchukene & -11.6 \\
4 & M00035859 & Palmarumycin CP1 & -11.4 \\
5 & M00002945 & Dehydrocycloguanandin & -11.3 \\
6 & M00006698 & Myrtillin & -11.0 \\
7 & M00001878 & Liriodenine & -10.9 \\
8 & M00005726 & Myricetin 3-alpha-l-arabinopyranoside & -10.8 \\
9 & M00005728 & Myricetin 3-galactoside & -10.7 \\
10 & M00025782 & Cassameridine & -10.7 \\
\hline
\end{tabular}

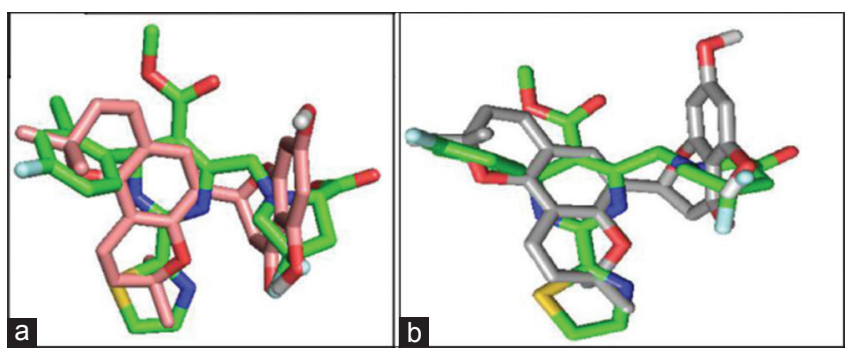

Fig. 3: (a) Superposition of docking AutoDock compound sanggenol 0 (pink) with 7TL (green) cocristal using PyMOL. (b) Superposition of docking AutoDock Vina compound sanggenol 0 (gray) with 7TL (green) cocristal using PyMOL 


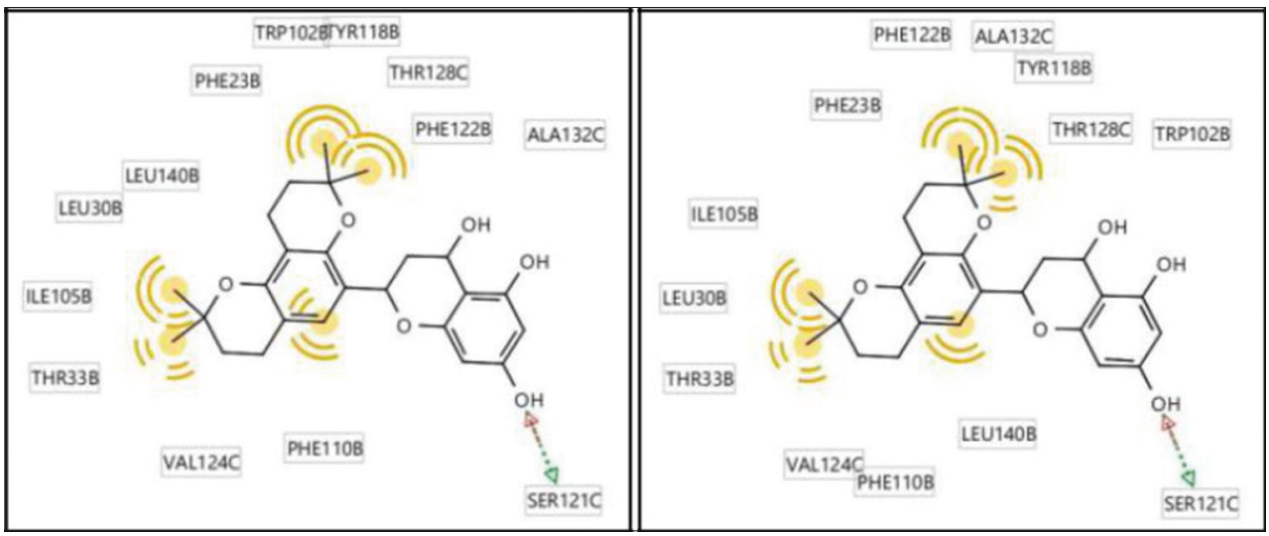

Fig. 4: Visualization of 2D interaction results of docking AutoDock (left) and AutoDock Vina (right) of sanggenol 0 compound using LigandScout

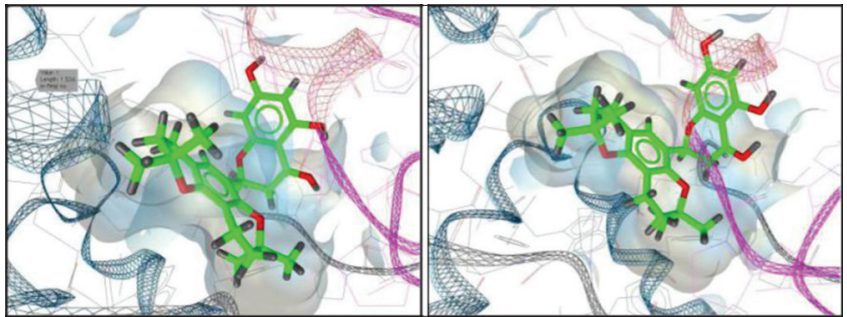

Fig. 5: Three-dimensional interaction visualization of AutoDock docking results (left) and Vina AutoDock (right) from sanggenol 0 compound using LigandScout. Blue band shows a B chain of macromolecules and the magenta-colored band shows the macromolecule C chain

that it decreased the degree of diclofenac-induced hepatic fibrosis in rats [12].

In the ninth position was the compound myricetin 3-galactoside, which can be obtained from the Camellia sinensis plant, known in Indonesia as tea. Tea has been shown to increase immunoglobulin (Ig) titer (IgM and $\operatorname{IgG}$ ) and to encourage the proliferation of lymphocytes in BALB/c rats given hepatitis $B$ vaccine [13]. In the tenth position was cassameridine compounds, which can be obtained from the plant Cassytha filiformis of the Lauraceae family. In traditional medicine, this plant is used to treat fever, influenza, and dysentery, and in experiments, it has shown antitrypanosomal activity [14].

Visualization and analysis of virtual screening results

The superposition visualization results showed that there was a structural similarity between the 7TL ligand and the virtual screening compound (Fig. 3). On the basis of the two-dimensional visualization results using LigandScout from the screening compound using AutoDock and AutoDock Vina, the amino acid residues that interact from the docking compound using AutoDock and AutoDock Vina were not very different as shown in Fig. 4. It was seen that the Sanggenol 0 compound for both the AutoDock and AutoDock Vina had the same interaction between the compound and the amino acid residue. In the ligands, a hydrophobic interaction was observed in residues of VAL 124C, THR 128C, ALA 132C, TYR, 118B, LEU 140B, TRP 102B, PHE 23B, THR 33B, ILE 105B, LEU 30B, PHE 122B, and PHE 110B. The -OH group in the compound exhibited an interaction as a hydrogen acceptor and a hydrogen donor on an SER $121 \mathrm{C}$ residue.

Three-dimensional visualization was performed to see the position of the ligand in tethering. Visualization between compounds with macromolecule was performed using LigandScout software (Fig. 5). On the basis of the visualization results, the ligand was seen interacting with the $\mathrm{B}$ chain and the macromolecule $\mathrm{C}$ chain and being on the binding pocket.

\section{CONCLUSION}

On the basis of our findings, it can be concluded that the validation of the method of virtual screening using AutoDock obtained the best AUC score with a value of 0.6709 and grid box with size $55 \times 55 \times 55$. Regarding the validation of virtual filtering methods using AutoDock Vina, the best AUC score was obtained with a value of 0.7832 and grid box size $20.625 \times 20.625 \times 20.625$. Virtual screening using AutoDock produced the following top 10 hit compounds: Yuehchukene, lansionic acid, stigmast-4-en-3-one, myrtillin, sanggenol 0, lanosterol, erycristagallin, alpha-spinasterol, cyanidin 3-arabinoside, and cathasterone. Virtual screening using AutoDock Vina produced the following top 10 hit compounds: Sanggenol O, cucumerin A, yuehchukene, palmarumycin CP1, dehydrocycloguanandin, myrtillin, liriodenine, myricetin 3-alphaL-arabinopyranoside, myricetin 3-galactoside, and cassameridine. Thus, three compounds were in the top lists of both virtual screening methods: myrtillin, sanggenol 0 , and yuehchukene have a possibility to be improve as a lead compound of anti HBV.

\section{ACKNOWLEDGMENT}

All authors would like to express gratitude toward Ministry of Research, Technology and Higher Education of Republic Indonesia for financial support through INSINAS Grant 2017.

\section{CONFLICTS OF INTEREST}

Authors declare no conflicts of interest in this research.

\section{REFERENCES}

1. Khan J, Alotaibi A. Study of hepatitis B virus infection and its genotypes in tribal people. Asian J Pharm Clin Res 2017;10:51-5.

2. Dinesh R, Ramalakshmi S. Prevalence of hepatitis B virus and associated risk factors in Irula tribal population. Asian J Pharm Clin Res 2017;10:100-2.

3. Li L, Chirapu SR, Finn MG, Zlotnick A. Phase diagrams map the properties of antiviral agents directed against hepatitis B virus core assembly. Antimicrob Agents Chemother 2013;57:1505-8.

4. Liu C, Fan G, Wang Z, Chen HS, Yin CC. Allosteric conformational changes of human HBV core protein transform its assembly. Sci Rep 2017;7:1404.

5. Yanuar, A. Molecular Docking of Practice and Application on Virtual Screening. Depok: Faculty of Pharmacy, Universitas Indonesia; 2012.

6. Liu T, Zhao J, Li H, Ma L. Evaluation on anti-hepatitis viral activity of Vitis vinifer $\mathrm{L}$. Molecules 2010;15:7415-22.

7. Unni SC, Soniya EV. Transgenic Cucumis sativus expressing the hepatitis B surface antigen. Plant Mol Biol Report 2010;28:627-34.

8. Sarkiyaki S, Zailani HA, Simon JG. Effects of aqueous stem bark extract of Jatropha curcas on some biochemical indices of mice infected with Plasmodium berghei. Am J Biochem 2016;6:130-5.

9. Zakaria MB, Vijayasekaran, Ilham Z, Muhammad NA. Antiinflammatory activity of Calophyllum inophyllum fruits extracts. Procedia Chem 2014;13:218-20 
10. Garg S, Sharma K, Ranjan R, Attri P, Mishra P. In vivo antioxidant activity and hepatoprotective effects of methanolic extract of Mesua ferrea linn. Int J Pharm Tech Res 2009;1:1692-6.

11. Zuhrotun A, Suganda AG, Wirasutisna KR, Wibowo MS. Isolation of bioactive compound of Michelia champaca L. Bark and its activity test using mechanism-based yeast bioassay. Asian J Pharm Clin Res 2016;9:158-61

12. Baravalia Y, Vaghasiya Y, Chanda S. Hepatoprotective effect of Woodfordia fruticosa kurz flowers on diclofenac sodium induced liver toxicity in rats. Asian Pac J Trop Med 2011;4:342-6.

13. Peranginangin JM, Sapatarini O, Ismawati E, Wihardy RA, Fadly R. Effect of steeped black tea (Camellia sinensis (L.) var. assamica) on immunoglobulin titer and lymphocyte proliferation in responses to hepatitis B vaccine in mice. Procedia Chem 2011;13:128-33.

14. Hoet S, Stévigny C, Block S, Opperdoes F, Colson P, Baldeyrou B, et al. Alkaloids from Cassytha filiformis and related aporphines: Antitrypanosomal activity, cytotoxicity, and interaction with DNA and topoisomerases. Planta Med 2004;70:407-13. 